\title{
Laminar flow inhibits TNF-induced ASK1 activation by preventing dissociation of ASK1 from its inhibitor 14-3-3
}

\author{
Yingmei Liu, Guoyong Yin, James Surapisitchat, Bradford C. Berk, and Wang Min \\ Center for Cardiovascular Research, University of Rochester Medical Center, Rochester, New York, USA \\ Address correspondence to: Wang Min, Center for Cardiovascular Research, University of Rochester Medical Center, \\ 601 Elmwood Avenue, Box 679, Rochester, New York 14642, USA. \\ Phone: (716) 273-1499; Fax: (716) 275-9895; E-mail: wang_min@urmc.rochester.edu. \\ Yingmei Liu and Guoyong Yin contributed equally to this work.
}

Received for publication December 6, 2000, and accepted in revised form January 30, 2001.

The inflammatory cytokine TNF- $\alpha$ stimulates several presumed pro-atherogenic signaling events in endothelial cells (ECs), including activation of c-Jun $\mathrm{NH}_{2}$-terminal kinase (JNK) and induction of E-selectin. Here, we show that apoptosis signal-regulating kinase 1 (ASK1), a MAP kinase kinase kinase, is required for TNF-mediated JNK activation. TNF activates ASK1 in part by dissociating ASK1 from its inhibitor 14-3-3. Because the risk of atherosclerosis is decreased in regions of steady laminar flow, we hypothesized that laminar flow inhibits proinflammatory cytokine-mediated activation of JNK. Steady laminar flow inhibited both TNF activation of ASK1 and JNK. Inhibition of ASK1 by flow correlated with increased association of ASK1 with 14-3-3. A constitutively active form of ASK1 lacking the 14-3-3-binding site (ASK1- $\triangle$ NS967A) was not inhibited by flow. These data establish ASK1 as a target for flow-mediated inhibition of cytokine signaling and indicate a novel role for 14-3-3 as an anti-inflammatory mediator in ECs.

J. Clin. Invest. 107:917-923 (2001).

\section{Introduction}

The c-Jun $\mathrm{NH}_{2}$-terminal kinase (JNK) is one of three mitogen-activated protein kinase (MAPK) cascades that are strongly activated by stress signals and proinflammatory cytokines such as TNF and IL-1. The TNF superfamily uses receptors that are devoid of intrinsic catalytic activity (1). Activation of JNK by members of the TNF-receptor family is thought to be mediated by a family of intracellular signaling molecules known as TNFR-associated factors (TRAFs) (2). Six TRAF family members have been described, and different members of this family display distinct receptor-binding specificities. Studies from TRAF2 transgenic and knockout mice demonstrated that TRAF2 is essential for activation of JNK in response to TNF but not IL-1 (3-5). In contrast, TRAF6 specifically mediates IL-1-induced JNK activation by IL-1 receptor $(6,7)$.

JNK is activated by dual phosphorylation mediated by one of the MAP2Ks (MKK4 and MKK6). MKK, in turn, is activated through phosphorylation by MAP3Ks (including MEKK1, TAK1, and ASK1) (8-10). Some of MAP3Ks such as MEKK1 and TAK1 can activate both $\mathrm{NF}-\mathrm{KB}$ and JNK cascades and these MAP3Ks are direct targets of TRAF molecules $(6,11,12)$. However, some MAP3Ks such as ASK1, appear to be involved in JNK activation only in response to proinflammatory cytokines and stress stimuli (13-15). ASK1 is a $170-\mathrm{kDa}$ protein that functionally is composed of an inhibitory $\mathrm{NH}_{2}$-terminal domain, an internal kinase domain, and a $\mathrm{COOH}$-terminal regulatory domain. The $\mathrm{COOH}$-terminal domain of ASK1 binds to the TRAF domain, and this association is required for ASK1 activation by TRAF2 and TRAF6 (15). A kinase-inactive form of ASK1 (ASK1-K709R) functions as a dominant negative in cytokine-induced JNK activation $(13,15)$. Deletion of the $\mathrm{NH}_{2}$-terminal 648 amino acids of ASK1 (ASK1- $\Delta \mathrm{N}$ ) leads to constitutive ASK1 kinase activity as it does in other MAP3Ks, indicating that the $\mathrm{NH}_{2}$-terminus contains an inhibitory domain $(16,17)$.

In addition, several cellular factors, 14-3-3, and thioredoxin, have been reported to inhibit ASK1 activity. Thioredoxin in a reduced form binds to the $\mathrm{NH}_{2}-$ terminal part of ASK1 and blocks activation of ASK1 by TNF (16-18); 14-3-3, a phosphoserine-binding molecule, binds to ASK1 specifically via Ser-967 of ASK1 and has been reported to inhibit ASK1-induced apoptosis (19). However, the role of 14-3-3 in TNF-induced ASK1 activation has not been determined.

It is widely accepted that inflammation plays a key role in the pathogenesis and progression of atherosclerosis (20). An important role for JNK in inflammation is supported by many studies. First, JNK is activated by almost all proinflammatory mediators such as TNF, IL-1, LPS, and oxidative stress $(10,21)$. Second, JNK activation is essential for expression of many proinflammatory molecules such as such as E-selectin, RANTES, IL-12, IL-6, and IL- 8 by activating transcription factors including cJun and ATF-2 (10, 22-24). This has been demonstrated by either a dominant-negative or antisense approach. For example, overexpression of DN-JNK or antisense JNK cDNA can block IL-6 and IL-8 expression induced by IL-1 (25); overexpression of DN-JNK can block 
endothelial cell (EC) surface expression in human umbilical vein endothelial cells (HUVECs) (23).

The concept that steady laminar blood flow exerts an atheroprotective effect by modulating EC function is well supported $(26,27)$. Examples include increased nitric oxide production and decreased expression of cell adhesion molecules such as VCAM-1 and E-selectin (26-28). In this study we demonstrate that activation of JNK, but not NF- $\mathrm{KB}$, by proinflammatory cytokines is attenuated by pre-exposure of EC to laminar flow. To gain insight into the mechanism by which laminar flow inhibits JNK activation by proinflammatory cytokines, we examined the effect of flow on the upstream activators of JNK in TNF signaling pathway. Our results show that flow inhibits ASK1 activity and ASK1dependent JNK activation by increasing association of ASK1 with its inhibitor 14-3-3.

\section{Methods}

Plasmids. Mammalian expression plasmids for Flag-epitope labeled wild-type TRAF2 and dominant-negative TRAF2 (dnTRAF2) (29) were provided by D.V. Goeddel (Tularik Inc., South San Francisco, California, USA); wild-type and the kinase-inactive ASK1 by Genhong Chen (University of California, Los Angeles, California, USA); GST-14-3-3 by Anthony J. Muslin (Washington University in St. Louis, St. Louis, Missouri, USA); GSTJNKK1 (MKK4) by Bing Su (M.D. Anderson, Texas, USA), pBIIXLUC plasmid (KB-LUC) (23), which contains two $\mathrm{KB}$ sites from the immunoglobulin kappa enhancer, and the JNK-dependent reporter gene system (FR-Luc and FA2-cJun) were from Stratagene (PathDetect Reporting Systems; La Jolla, California, USA). Expression plasmids for ASK1- $\Delta \mathrm{N}$ (lacking the $\mathrm{NH}_{2}-$ terminal domain) and ASK1- $\triangle$ NS967A (deletion of the $\mathrm{NH}_{2}$-terminal and mutation at serine 967) were constructed into the Flag-vector.

Cells and cytokines. HUVECs are purchased from Clonetics Corp. (San Diego, California, USA). Human rTNF and rIL-1 are from R\&D Systems (Minneapolis, Minnesota, USA) and used at $100 \mathrm{U} / \mathrm{ml}$ and $250 \mathrm{U} / \mathrm{ml}$, respectively.

Flow experiments. Fluid shear stress in vitro was created by the cone and plate viscometer. Cells were grown on $60-\mathrm{mm}$ dishes coated with gelatin. Upon reaching $95 \%$ confluence, fresh media was added, and 2 days later cells were rinsed free of culture media with HBSS (containing, in mM: NaCl 130, KCl 5, $\mathrm{CaCl}_{2} 1.5, \mathrm{MgCl}_{2}$ 1.0, HEPES 20, pH 7.4), with $10 \mathrm{mM}$ glucose added, and $10 \%$ serum, and either maintained in static condition or exposed to flow (fluid shear stress $=12$ dynes $/ \mathrm{cm}^{2}$ ) in a parallel plate chamber at $37^{\circ} \mathrm{C}$. After varying times of exposure to flow, cells were washed gently with icecold PBS (composition, in $\mathrm{mM}: \mathrm{NaCl} 137, \mathrm{KCl} 2.7$, $\mathrm{Na}_{2} \mathrm{HPO}_{4} 4.3, \mathrm{KH}_{2} \mathrm{PO}_{4}$ 1.4, $\mathrm{pH}$ 7.3) and cell lysates were for further analysis.

JNK and ASK1 kinase assays. JNK assay was performed as described previously (23) using GST-c-Jun (1-80) fusion protein as a substrate. ASK1 assay was performed using GST-MKK4 as a substrate.
Transfection and reporter assay. Transfection of HUVECs were performed by DEAE-dextran method, as described previously (23). Luciferase activity followed by renilla activity was measured twice in duplicate using a Berthold luminometer. All data were normalized as relative luciferase light units/renilla unit.

Immunoprecipitation and immunoblotting. HUVECs after various treatments were washed twice with cold PBS and lysed in $1.5 \mathrm{ml}$ of cold lysis buffer $(50 \mathrm{mM}$ Tris-HCl, pH 7.6, $150 \mathrm{mM} \mathrm{NaCl}, 0.1 \%$ Triton X-100, $0.75 \%$ Brij $96,1 \mathrm{mM}$ sodium orthovanadate, $1 \mathrm{mM}$ sodium fluoride, $1 \mathrm{mM}$ sodium pyrophosphate, 10 $\mu \mathrm{g} / \mathrm{ml}$ aprotinin, $10 \mu \mathrm{g} / \mathrm{ml}$ leupeptin, $2 \mathrm{mM}$ PMSF, 1 mM EDTA) for 20 minutes on ice. For immunoprecipitation to analyze protein interaction in vivo, supernatant of cell lysates were precleared by incubating with normal rabbit serum plus GammaBind plus Sepharose beads on a rotator at $4^{\circ} \mathrm{C}$ overnight. The lysates were then incubated with the first protein-specific antiserum (e.g., 14-3-3; Santa Cruz Biotechnology Inc., Santa Cruz, California, USA) for 2 hours with $50 \mu \mathrm{l}$ of GammaBind plus Sepharose. Immune complexes were collected after each immunoprecipitation by centrifugation at $13,000 \mathrm{~g}$ for 10 minutes, followed by three to five washes with lysis buffer. The immune complexes were subjected to SDS-PAGE followed by immunoblot analysis (Immobilon P; Millipore, Bedford, Massachusetts, USA) with the second proteinspecific Ab (e.g., ASK1; Santa Cruz Biotechnology Inc.). The chemiluminescence was detected using an enhanced chemiluminescence (ECL) kit according to the instructions of the manufacturer (Amersham Life Science, Arlington Heights, Illinois, USA). For detection of Flag-tagged proteins (ASK1- $\Delta \mathrm{N}$ and ASK1$\triangle$ NS967A), anti-Flag M2 Ab (Sigma, St. Louis, Missouri, USA) was used for immunoblot analysis. For detection of HA-tagged proteins (wild-type ASK1), anti-HA Ab (Roche Diagnostics, Indianapolis, Indiana, USA) was used for immunoblot analysis.

Electrophoretic mobility shift assays. The double-stranded oligonucleotide containing a $\mathrm{KB}$ consensus site from the immunoglobulin $\kappa$ gene (Promega Corp., Madison, Wisconsin, USA) was used for electrophoretic mobility shift assays (EMSAs). Preparation of nuclear extracts and EMSA were performed as described previously (23).

\section{Results}

Flow inhibits JNK and ASK1 activation induced by cytokines. To examine the effect of flow on cytokine-induced JNK activation, HUVECs were either maintained in static conditions for 10 minutes or subjected to flow (shear stress $=12$ dynes $\left./ \mathrm{cm}^{2}\right)$ for 10 minutes before TNF- $\alpha(100$ $\mathrm{U} / \mathrm{ml}$ ) stimulation for 15 minutes. TNF-induced JNK activation was determined by an in vitro kinase assay using GST-c-Jun as a substrate, as described previously (23). The results show that pre-exposure to flow significantly attenuated TNF-induced JNK activation. JNK activity was also measured by determining phosphorylation of endogenous c-Jun using Western blot analysis 


\section{Figure 1}

Flow pre-exposure inhibits TNF-mediated JNK and ASK1, but not NF$\kappa B$, activation in ECs. ECs were subjected to the following "preconditioning" protocol: they were maintained in static conditions for 25 minutes (Ctrl), exposed to flow for 10 minutes and then held static for 15 minutes (Flow), maintained in static conditions for $10 \mathrm{~min}$ utes, followed by TNF stimulation for 15 minutes or subjected to flow for 10 minutes followed by TNF- $\alpha(100 \mathrm{U} / \mathrm{ml})$ stimulation for 15 minutes (flow + TNF). (a) Flow inhibits JNK activation by TNF. Cell lysates were prepared and analyzed for JNK activity by an in vitro kinase assay using GST-c-Jun as a substrate. (b) Flow has no inhibitory effect on NF-KB. Cells were treated as in $\mathbf{a}$. Nuclear extracts from these cells were used for EMSA with a $\kappa B$ probe. Specificity of $\mathrm{NF}-\kappa \mathrm{B}$ complex was verified by 50 -fold molar excess of the unlabeled $\kappa B$ oligonucleotide in the TNF-treated sample (TNF + competitor). (c) Flow inhibits ASK1 activation by TNF. Cell lysates in a were prepared and analyzed for ASK1 activity by an in vitro kinase assay using GST-MKK 4 as a substrate. Autoradiograms shown in $\mathbf{a}-\mathbf{c}$ are representative of three experiments in HUVECs.

with an $\mathrm{Ab}$ against phosphorylated c-Jun (23). A similar effect of flow on TNF-induced JNK activation was observed by this assay. In contrast to our findings, two other laboratories $(30,31)$ observed JNK activation by flow. "Trivial" explanations for the differences include: we used HUVECs rather than bovine aortic endothelial cells; our cells were several days postconfluent; and our cells were growth-arrested by contact inhibition while the other laboratories used serum deprivation.

To examine whether flow specifically inhibits JNK activation by TNF, we determined the effect of flow on NF-KB activation, a signaling pathway activated in parallel to JNK. We have shown previously that TNF activates main-

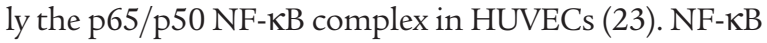
activation was measured by an EMSA using HUVEC nuclear extracts. As shown in Figure 1b, pre-exposing HUVECs to flow did not inhibit TNF-stimulated NF-кB activation. These data indicate that flow specifically inhibits JNK activation by proinflammatory cytokines.

\section{Figure 2}

ASK1 is specifically involved in JNK activation by TNF. HUVECs were transiently transfected with the indicated expression constructs ( $1 \mu \mathrm{g}$ each) together with either JNK (a) or a NF-KB (b) promoter-reporter gene ( $1 \mu \mathrm{g}$ each). A constitutive expression vector for renilla unit $(0.5 \mu \mathrm{g}$ each) was also transfected for normalization of transfection efficiency (see Methods). Cells were left untreated or treated with TNF $(100 \mathrm{U} / \mathrm{ml} \mathrm{TNF-} \alpha)$. Relative luciferase activities (luciferase vs. renilla unit) from untreated or TNF-treated samples are presented from the mean of duplicate samples. Similar results were obtained from two additional experiments. The TNF responses (fold induction) are shown. (a) Effect of kinase-inactive ASK1 (ASK1-K709R) or DN-TRAF2 on a JNK-dependent reporter gene. (b) Effect of kinase-inactive ASK1 (ASK1-K709R) or DN-TRAF2 on a NF-KB-dependent reporter gene. a

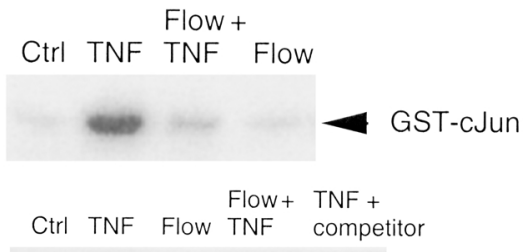

b

c
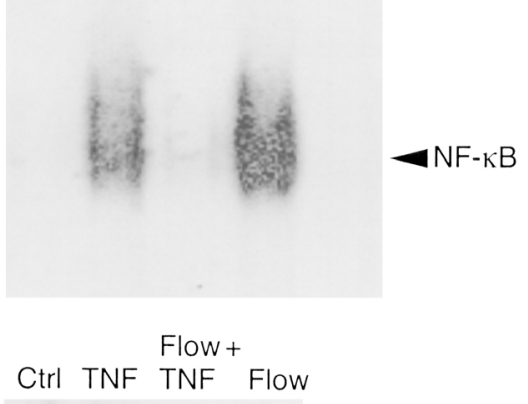

GST-MKK4
To examine the effect of flow on upstream activators of JNK, we measured the activity of ASK1, one of the MAP3Ks involved in activation of JNK, but not NF- $\kappa B$. ASK1 activity was determined by an in vitro kinase assay using GST-MKK4 (JNKK1) fusion protein as a substrate. As shown in Figure 1c, TNF- $\alpha$ (100 U/mL) activated ASK1 maximally at 15 minutes in HUVECs. Pre-exposing HUVECs to flow for 10 minutes significantly inhibited TNF-stimulated ASK1 activity $(80 \%$ inhibition; $n=3, P<0.01$ ).

ASK1 is specifically involved in JNK activation by TNF. To examine if ASK1 is a specific activator in JNK pathway in HUVECs, we performed JNK- and NF-KB-dependent reporter gene assays by transient transfection in ECs. We used TRAF2 as a control, since we found previously that TRAF2 is involved in TNF-induced activation of both NF- $K B$ and JNK in ECs $(23,32)$. Overexpression of dominant-negative TRAF2 (DN-TRAF2) blocked TNFinduced expression of JNK-dependent reporter genes
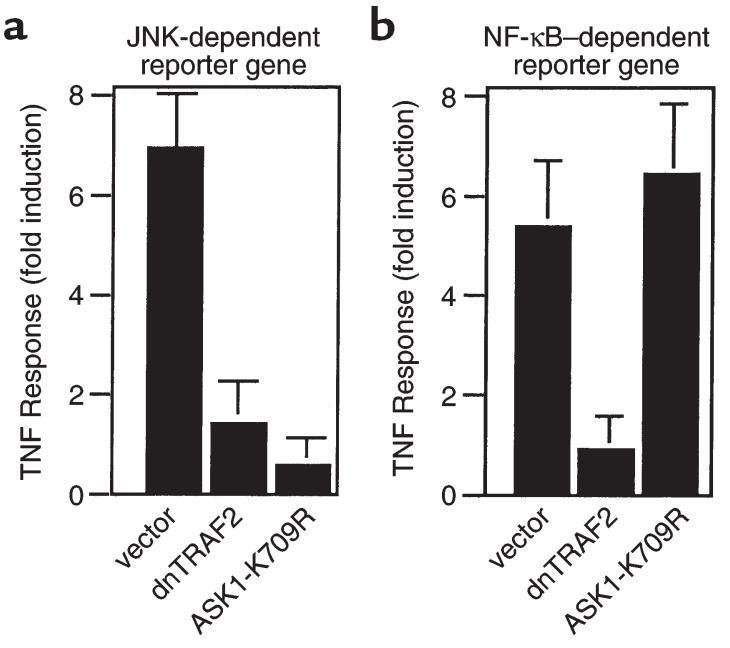


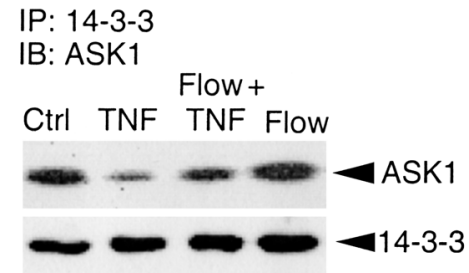

\section{Figure 3}

Flow enhances the interaction of ASK1 with its inhibitor 14-3-3. HUVECs were subjected to flow and TNF treatment as described in Figure 1. Cell lysates were immunoprecipitated (IP) with Ab against 14-3-3 followed by an immunoblot (IB) analysis with anti-ASK1 (top panel). The 14-3-3 protein in the immunoprecipitates was determined by Western blot analysis with Ab against 14-3-3 (lower panel).

(Figure 2a) and NF-אB-dependent gene expression (Figure $2 \mathrm{~b}$ ). In contrast, overexpression of kinase-inactive ASK1 dominant negative (ASK1-K709R) blocked both TNF-induced expression of JNK-dependent reporter genes (Figure 2a), but not NF-KB-dependent gene expression (Figure 2b). These data suggest that ASK1 is specifically required for JNK activation by TNF.

TNF dissociates ASK1 from its inbibitor 14-3-3, whereas flow prevents dissociation of ASK1 from 14-3-3. We hypothesized that flow increases the interaction of ASK1 with its inhibitor 14-3-3 to explain how ASK1 activation by TNF is inhibited by flow. Association of ASK1 with 14-3-3 was easily detected in untreated HUVECs (Figure 3 , control). TNF treatment significantly reduced the interaction of ASK1 with 14-3-3, indicating that TNF activates ASK1, in part, by dissociating ASK1 from 14-3-3. In contrast, flow (using the preconditioning protocol) prevented the TNF-induced dissociation of ASK1 from 14-3-3 (Figure 3).

ASK1- 1 NS967A does not associate with 14-3-3. While thioredoxin binds to the $\mathrm{NH}_{2}$-terminal domain of ASK1 and 14-3-3 binds to the COOH-terminal of ASK1, both inhibit ASK1 activity. An $\mathrm{NH}_{2}$-terminal ASK1 deletion mutant (ASK1- $\Delta \mathrm{N}$ ) no longer binds to thioredoxin and shows increased kinase activity (16, 17). Mutation of serine $967 \rightarrow$ alanine (ASK1-S967A) renders ASK1 defective in 14-3-3 binding and likely increases ASK1 activity as shown by accelerated ASK1induced apoptosis (19). We constructed an ASK1 "double" mutant, which lacks the $\mathrm{NH}_{2}$-terminal domain and has a mutation of serine $967 \rightarrow$ alanine that we termed ASK1- NS967A. To determine whether ASK1- $\triangle$ NS967A binds to these two inhibitors, HA-tagged ASK1-WT, Flag-tagged ASK1$\Delta \mathrm{N}$ and ASK1- $\Delta$ NS967A were transiently transfected into HUVECs, and the interactions of these ASK1 proteins with thioredoxin and 14-3-3 were examined by coimmunoprecipitation assay. As expected, ASK1-WT bound to both thioredoxin and 14-3-3 (Figure 4a). ASK1- $\Delta \mathrm{N}$ bound to 14-3-3 (lane 1 in Figure $4 \mathrm{~b}$ ), but not to thioredoxin (lane 3 in Figure $4 \mathrm{~b}$ ). In contrast, ASK1- $\triangle$ NS967A failed to bind either thioredoxin or 14-3-3 (lanes 2 and 4 in Figure 4b).
ASK1- $\triangle N S 967 A$ activity is not inbibited by flow. To determine the effect of altering ASK1 interaction with 14-3-3, we measured both JNK activity and JNK reporter-gene expression. Expression of ASK1 proteins in transfected ECs was determined by Western blot analysis with an $\mathrm{Ab}$ against the $\mathrm{COOH}$-terminal ASK1, which recognized ASK1-WT, ASK1-ASK1K709R, ASK1- $\Delta$ N, and ASK1- $\Delta$ NS967A (Figure 5a). The endogenous ASK1 present in ECs was also detected (see lanes 4 and 5). Expression of ASK1-WT, ASK1$\Delta \mathrm{N}$, or ASK1- $\Delta$ NS967A increased JNK activity in the absence of TNF as measured by an in vitro kinase assay with GST-c-Jun substrate (Figure 5b) and JNKdependent reporter-gene expression (Figure $5 \mathrm{c}$ ). Expression of the kinase-inactive form of ASK1 (ASK1-K709R) had no effect on JNK reporter-gene expression (Figure $5 \mathrm{c}$ ). These data confirm the function of ASK1 protein in ECs.

Since ASK1- $\triangle$ NS967A does not associate with 14-3-3, we hypothesized that ASK1- $\triangle$ NS967A activity would not be inhibited by flow. To test this hypothesis, ECs were transfected with Flag-tagged ASK1- $\triangle \mathrm{N}$ or ASK1$\triangle$ NS967A constructs. Because ASK1- $\Delta \mathrm{N}$ and ASK1$\triangle$ NS967A are constitutively active, ECs were exposed to flow in the presence of TNF 24 hours after transfection.

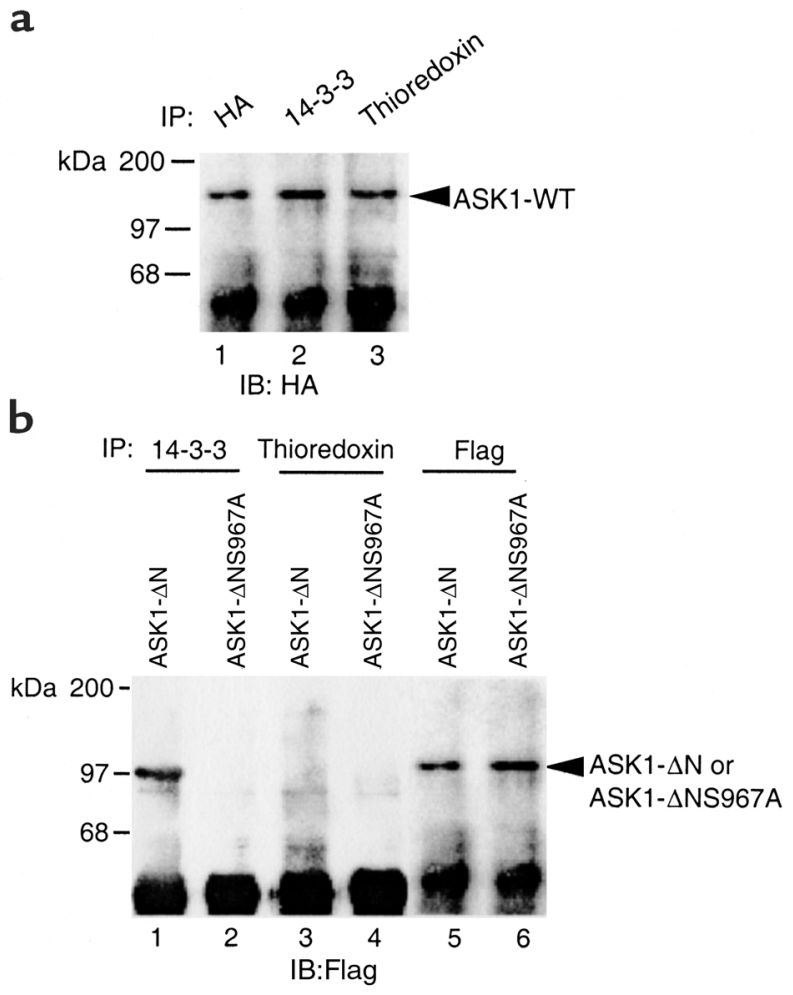

\section{Figure 4}

ASK1- $\triangle$ NS967A does not bind either thioredoxin or 14-3-3. HAtagged ASK1-WT, Flag-tagged ASK1- $\Delta \mathrm{N}$ or ASK1- $\Delta$ NS967A were transiently transfected into HUVECs. Cell lysates were immunoprecipitated by Ab's as indicated (HA, 14-3-3, thioredoxin, or Flag). ASK1-WT was detected by Western blot analysis with anti-HA (a). ASK1- $\Delta \mathrm{N}$ and ASK1- $\mathrm{N}$ S967A were detected by anti-Flag (b). 
a

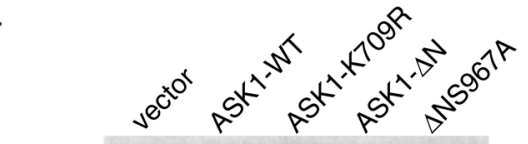

kDa 200-

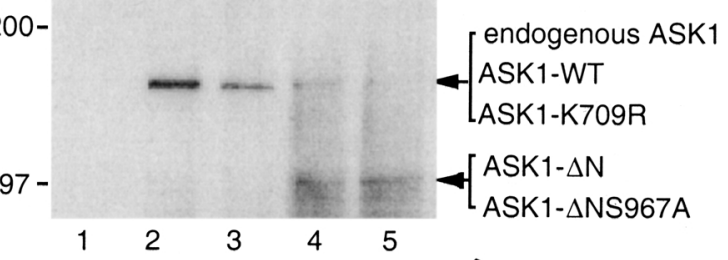

$97-$

b

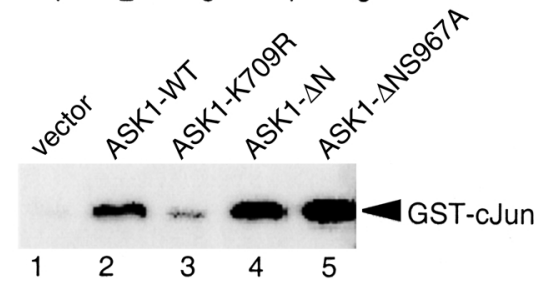

C

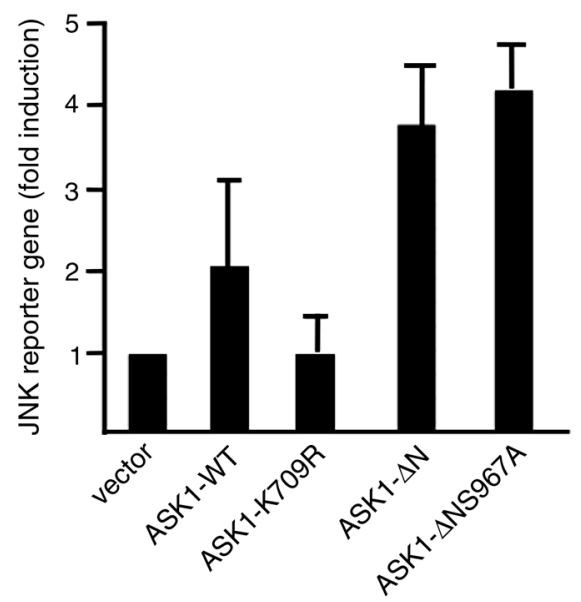

Figure 5

ASK1- $\Delta$ N and ASK1- $\Delta$ NS967A show increased JNK activation. (a) Overexpression of ASK1 proteins in ECs. HUVECs were transiently transfected with control vector, ASK1-WT, ASK1-K709R, ASK1- $\Delta \mathrm{N}$, and ASK1- 1 NS967A, and cell lysates were used for protein expression by Western blot with $\mathrm{Ab}$ against the $\mathrm{COOH}$-terminal domain of ASK1. (b) Effect of ASK1 proteins on JNK activation. Cell extracts in a were used for in vitro kinase assay using GST-c-Jun as a substrate. (c) Effects of ASK1 proteins on JNK-dependent promoter. HUVECs were cotransfected with $1 \mu \mathrm{g}$ of JNK reporter gene, a construct for renilla unit $(0.5 \mu \mathrm{g})$, and various ASK1 expression constructs $(1 \mu \mathrm{g}$ each). The relative luciferase activity normalized to renilla unit is shown. Data are presented from mean of duplicate samples.

Flag-tagged ASK1- $\Delta \mathrm{N}$ or ASK1- $\Delta$ NS967A were immunoprecipitated by anti-Flag, and their activities were determined by an in vitro kinase assay using GST-MKK4 as a substrate. As shown in Figure 6a, ASK1- $\Delta \mathrm{N}$ expression was not altered by flow, but its activity, as measured by phosphorylation of GST-MKK4, was significantly inhibited by flow (Figure 6, b and c). In contrast, the activity of ASK1- $\triangle$ NS967A was not inhibited by flow. These data suggest that flow inhibits ASK1 activity by regulating association of ASK1 with its inhibitor 14-3-3.

Flow and TNF modify phosphorylation of ASK-1 Ser-967. Ser-967 of ASK1 is part of a motif RxSxxP reported to bind 14-3-3; therefore, we hypothesized that TNF and a

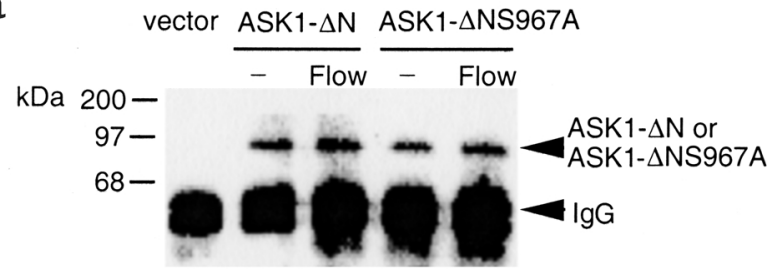

b

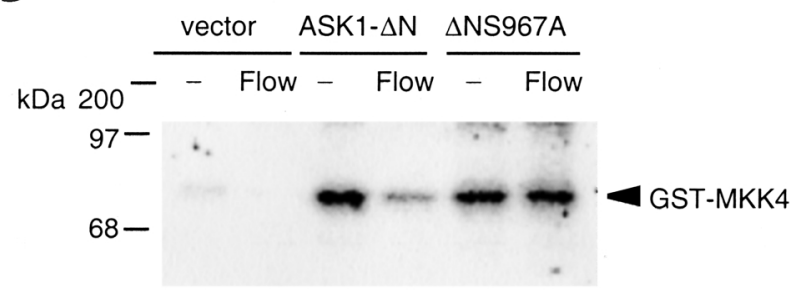

C

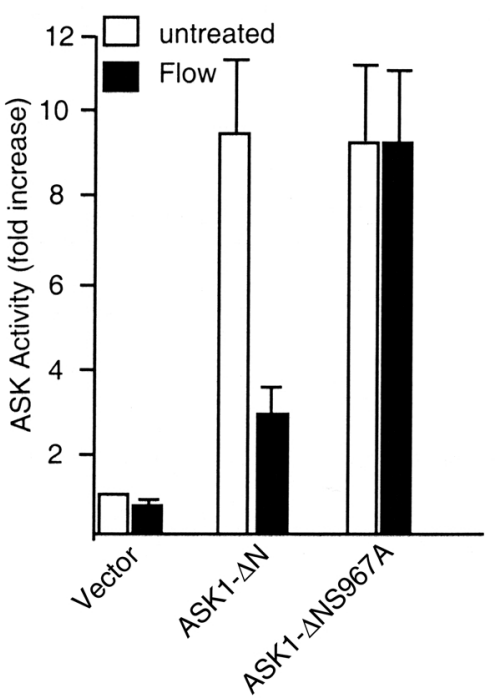

\section{Figure 6}

ASK1- $\triangle$ NS967A activity is not inhibited by flow. HUVECs were transfected with control vector, ASK- $\Delta \mathrm{N}$, or ASK1- $\Delta$ NS967A. Twenty-four hours after transfection, cells were subjected to "preconditioning" protocol. Cell lysates were immunoprecipitated by anti-Flag. The immunoprecipitates were used to detect ASK1 by Western blot analysis with anti-Flag for protein expression (a) and to determine ASK1 activity by an in vitro kinase assay using GST-MKK4 as a substrate (b). (c) The quantitative analysis of the radiogram in b. ASK1 activity is shown as fold increase by taking vector transfection as one. Data are presented from mean of two independent experiments.

flow alter ASK1 phosphorylation at Ser-967 to regulate the interaction of ASK1 with 14-3-3 (Figure 7a). To test this hypothesis, HA-ASK1 transfected into ECs. ECs were exposed to flow or TNF 24 hours later. To measure pSer-967 of ASK1 we developed an in vitro 14-3-3 binding assay. Phosphorylated ASK1 Ser-967 was first bound to GST-14-3-3, followed by Western blot analysis using anti-HA. This is different from the coimmunoprecipitation assay described in Figure 3, which measured preexisting complexes of ASK1 and 14-3-3. We first examined function of GST-14-3-3 proteins. The results show that ASK1 specifically binds to wildtype 14-3-3, but not to a mutant 14-3-3 (K49A) defec- 
tive in phosphoserine-binding (33) (Figure 7b). Consistent with our observation in Figure 4, ASK1- $\Delta$ N, but not ASK1- $\triangle$ NS967A, bound to GST-14-3-3 (data not shown). These data suggest GST-14-3-3 specifically binds to phosphorylated ASK1 Ser-967 in an vitro binding assay. Therefore, we use this GST-14-3-3 binding assay to determine effects of TNF and flow on phosphorylation of ASK1 Ser-967. Our results show that TNF decreased the amount of phosphorylated ASK1 leading to reduced binding on GST-14-3-3 (Figure $7 \mathrm{~b}$ ). In contrast, flow prevents dephosphorylation of ASK1 at Ser-967, resulting in increased 14-3-3 binding (Figure 7c). These data suggest that TNF and flow regulate ASK1 activity by modifying the phosphorylation status of ASK1 Ser-967.

\section{Discussion}

The major finding of the present study is that one of the mechanisms by which steady laminar flow inhibits proinflammatory events is via inhibition of ASK1. Based on findings in the present study, we propose a model in which TNF activates ASK1 (in part) by disso-

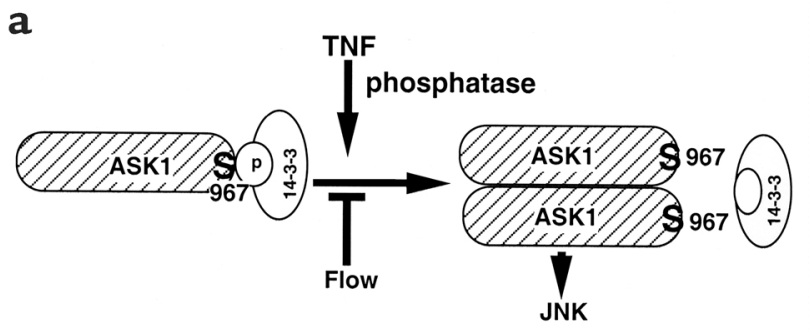

b

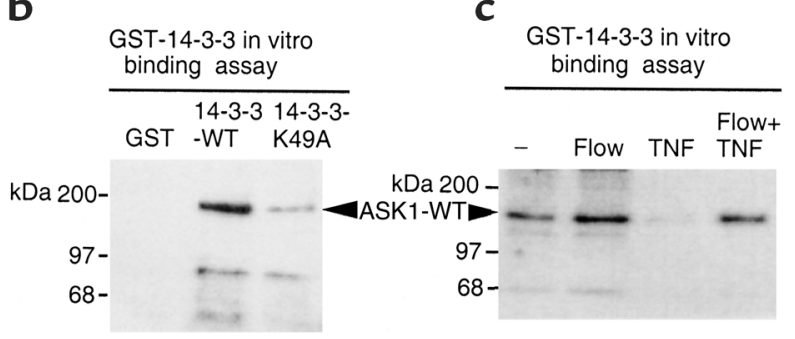

\footnotetext{
Figure 7

Flow and TNF modify phosphorylation of ASK1 Ser-967. (a) A model for regulation of ASK1 activity by TNF and flow. ASK1 Ser-967 is in a phosphorylated state (pSer-967). TNF induces dephosphorylation of pSer-967 to release ASK1 from inhibitor 14-3-3. ASK1 dimerization results in activation of the ASK1-JNK cascade. Flow prevents TNF-induced dephosphorylation of pSer-967 to block release of ASK1 from 14-3-3. (b) GST-14-3-3 binding assay for phosphorylation of ASK1 Ser-967. HA-tagged ASK1-WT was transfected into ECs, and pSer-967 of ASK1 was determined by an in vitro binding to GST-14-3-3 followed by Western blot analysis using anti-HA. GST, GST-14-3-3-K49A (a phosphoserine binding-deficient mutant) were used as controls. (c) Flow and TNF regulate phosphorylation of ASK1 Ser-967. ECs were transfected with ASK-WT. Twenty-four hours after transfection, cells were subjected to "preconditioning" protocol. Amount of phosphorylated ASK1 Ser-967 was measured by an in vitro GST-14-3-3 binding assay. Data are representative of two experiments in HUVECs.
}

ciating ASK1 from its inhibitor 14-3-3, while steady laminar flow inhibits TNF-induced ASK1 and JNK activation by preventing the release of ASK1 from 14-3-3 (Figure 7). Because steady flow may exert atheroprotective effects by inhibiting inflammation, these results imply an important role for 14-3-3 in atherosclerosis.

While some MAP3Ks such as TAK1 and MEKK1 are involved in both JNK and NF- $\mathrm{KB}$ activation, our data indicate ASK1 is one of the major MAP3Ks specifically associated with JNK activation in ECs. It remains to be determined whether other MAP3Ks are regulated by TNF and flow in ECs.

The activation mechanisms for MAP3Ks in "the stress-activated" MAPK pathways remain largely unknown. A role for reactive oxygen species (ROS) has been proposed (17). Specifically, hydrogen peroxide activates ASK1, and TNF-induced activation of ASK1 is inhibited by antioxidants (18). Thioredoxin, a redoxsensing protein, associates with ASK1 in its reduced form. Recently, it has been shown that TNF (as well as overexpression of TRAF2) stimulates the production of ROS (16). Activation of ASK1 by TNF requires the ROSmediated dissociation of thioredoxin followed by binding of TRAF2 and subsequent ASK1 dimerization (16). The fact that flow also increases ROS in ECs $(34,35)$ suggests that thioredoxin is not a critical mediator of flow in regulating ASK1 activity. This is supported by our data that activity of the thioredoxin binding-deficient mutant (ASK1- $\Delta \mathrm{N}$ ) is still inhibited by flow.

We found that 14-3-3 binds to ASK1 in unstimulated HUVECs, indicating that ASK1 is constitutively phosphorylated at Ser-967 and forms a preexisting complex with 14-3-3 as described previously (19). A 14-3-3 molecule with two mutations (R56A and R60A) is deficient in its ability to bind phosphoserine-containing peptides and functions as a dominant-negative 14-3-3 signaling molecule (33). Recently Xing et al. showed that expression of DN-14-3-3 (R56A/R60A) activated ASK1 and JNK, likely by releasing ASK1 from inhibition by endogenous 14-3-3 (36). This is consistent with our finding that proinflammatory cytokines stimulate release of ASK1 from 14-3-3, whereas flow inhibits the release. Currently the mechanism by which cytokines and flow regulate association of ASK1 with 14-3-3 is not understood. However, our data suggest that phosphorylation of ASK1 Ser-967 (which is the 14-3-3 binding site) is the critical step. We suggest that TNF activates a phosphatase that diminishes 14-3-3-ASK1 interaction, whereas flow inhibits this phosphatase to enhance formation of 14-3-3-ASK1 complex (Figure 7). Our finding that activity of the 14-3-3 binding-deficient mutant (ASK1- $\mathrm{NS} 967 \mathrm{~A}$ ) is not inhibited by flow further supports this model. Experiments to identify this phosphatase are underway.

In summary, these data establish the ASK1-JNK signaling cascade as a target for flow-mediated inhibition of proinflammatory cytokine signaling and indicate a novel role for 14-3-3 as an anti-inflammatory mediator. While the roles of ASK1 and JNK in atherogenesis have 
only been studied to a limited extent, this study suggests that inhibition of ASK1-JNK pathway may provide a valid approach for antiatherosclerotic therapy.

\section{Acknowledgments}

We thank D.V. Goeddel for TRAF2 and DN-TARF2 constructs; Genhong Chen for wild-type and the kinaseinactive ASK1; Anthony J. Muslin for GST-14-3-3; Stephanie Lehoux for GST-14-3-3 K49A; Bing Su for GST-JNKK1 (MKK4). We also thank B.C. Berk lab members for assistance and discussions. This work was supported by NIH grants 1R01HL-65978-01 to W. Min and 5P01HL-18645 to B.C. Berk.

1. Baker, S.J., and Reddy, E.P. 1998. Modulation of life and death by the TNF receptor superfamily. Oncogene. 17:3261-3270.

2. Arch, R.H., Gedrich, R.W., and Thompson, C.B. 1998. Tumor necrosis factor receptor-associated factors (TRAFs): a family of adapter proteins that regulates life and death. Genes Dev. 12:2821-2830.

3. Lee, S.Y., et al. 1997. TRAF2 is essential for JNK but not NF-kappaB activation and regulates lymphocyte proliferation and survival. Immunity. 7:703-713.

4. Lomaga, M.A., et al. 1999. TRAF6 deficiency results in osteopetrosis and defective interleukin-1, CD40, and LPS signaling. Genes Dev. 13:1015-1024

5. Yeh, W.C., et al. 1997. Early lethality, functional NF-kappaB activation, and increased sensitivity to TNF-induced cell death in TRAF2-deficient mice. Immunity. 7:715-725.

6. Baud, V., et al. 1999. Signaling by proinflammatory cytokines: oligomerization of TRAF2 and TRAF6 is sufficient for JNK and IKK activation and target gene induction via an amino-terminal effector domain. Genes Dev. 13:1297-1308.

7. Cao, Z., Xiong, J., Takeuchi, M., Kurama, T., and Goeddel, D.V. 1996. TRAF6 is a signal transducer for interleukin-1. Nature. 383:443-446.

8. Derijard, B., et al. 1994. JNK1: a protein kinase stimulated by UV light and Ha-Ras that binds and phosphorylates the c-Jun activation domain. Cell. 76:1025-1037.

9. Ichijo, H. 1999. From receptors to stress-activated MAP kinases. Oncogene. 18:6087-6093.

10. Ip, Y.T., and Davis, R.J. 1998. Signal transduction by the c-Jun N-terminal kinase (JNK): from inflammation to development. Curr. Opin. Cell Biol. 10:205-219.

11. Lee, F.S., Hagler, J., Chen, Z.J., and Maniatis, T. 1997. Activation of the IkappaB alpha kinase complex by MEKK1, a kinase of the JNK pathway. Cell. 88:213-222.

12. Ninomiya-Tsuji, J., et al. 1999. The kinase TAK1 can activate the NIK I kappaB as well as the MAP kinase cascade in the IL-1 signalling pathway. Nature. 398:252-256.

13. Chang, H.Y., Nishitoh, H., Yang, X., Ichijo, H., and Baltimore, D. 1998. Activation of apoptosis signal-regulating kinase 1 (ASK1) by the adapter protein Daxx. Science. 281:1860-1863.

14. Ichijo, H., et al. 1997. Induction of apoptosis by ASK1, a mammalian MAPKKK that activates SAPK/JNK and p38 signaling pathways. Science. 275:90-94.

15. Nishitoh, H., et al. 1998. ASK1 is essential for JNK/SAPK activation by TRAF2. Mol. Cell. 2:389-395.

16. Liu, H., Nishitoh, H., Ichijo, H., and Kyriakis, J.M. 2000. Activation of apoptosis signal-regulating kinase 1 (ASK1) by tumor necrosis factor receptor-associated factor 2 requires prior dissociation of the ASK1 inhibitor thioredoxin. Mol. Cell. Biol. 20:2198-2208.

17. Saitoh, M., et al. 1998. Mammalian thioredoxin is a direct inhibitor of apoptosis signal-regulating kinase (ASK) 1. EMBO J. 17:2596-2606.

18. Gotoh, Y., and Cooper, J.A. 1998. Reactive oxygen species- and dimerization-induced activation of apoptosis signal-regulating kinase 1 in tumor necrosis factor-alpha signal transduction. J. Biol. Chem. 273:17477-17482.

19. Zhang, L., Chen, J., and Fu, H. 1999. Suppression of apoptosis signalregulating kinase 1 -induced cell death by 14-3-3 proteins. Proc. Natl. Acad. Sci. USA. 96:8511-8515.

20. Ross, R. 1999. Atherosclerosis: an inflammatory disease. N. Engl. J. Med. 340:115-126.

21. Force, T., Pombo, C.M., Avruch, J.A., Bonventre, J.V., and Kyriakis, J.M. 1996. Stress-activated protein kinases in cardiovascular disease. Circ. Res. 78:947-953.

22. Lu, H.T., et al. 1999. Defective IL-12 production in mitogen-activated protein (MAP) kinase kinase 3 (Mkk3)-deficient mice. EMBO J. 18: $1845-1857$

23. Min, W., and Pober, J.S. 1997. TNF initiates E-selectin transcription in human endothelial cells through parallel TRAF-NF-kappa B and TRAF-RAC/CDC42-JNK-c-Jun/ATF2 pathways. J. Immunol. 159:3508-3518.

24. Reed, M.A., et al. 1997. Tumor necrosis factor-a-induced E-selectin expression is activated by the nuclear factor-kB and c-Jun $\mathrm{N}$-terminal kinase/p38 mitogen-activated protein kinase pathways. J. Biol. Chem. 272:2753-2761.

25. Krause, A., et al. 1998. Stress-activated protein kinase/Jun N-terminal kinase is required for interleukin (IL)-1-induced IL-6 and IL-8 gene expression in the human epidermal carcinoma cell line KB. J. Biol. Chem. 273:23681-23689.

26. Davies, P.F. 1995. Flow-mediated endothelial mechanotransduction. Physiol. Rev. 75:519-560.

27. Traub, O., and Berk, B.C. 1998. Laminar shear stress: mechanisms by which endothelial cells transduce an atheroprotective force. Arterioscler. Thromb. Vasc. Biol. 18:677-685.

28. Gimbrone, M.A., Jr. 1999. Vascular endothelium, hemodynamic forces, and atherogenesis. Am. J. Pathol. 155:1-5.

29. Rothe, M., Sarma, V., Dixit, V.M., and Goeddel, D.V. 1995. TRAF2mediated activation of NF-kappa B by TNF receptor 2 and CD40. Science. 269:1424-1427.

30. Li, Y.S., et al. 1996. The Ras-JNK pathway is involved in shear-induced gene expression. Mol. Cell. Biol. 16:5947-5954.

31. Jo, H., et al. 1997. Differential effect of shear stress on extracellular signal-regulated kinase and $\mathrm{N}$-terminal Jun kinase in endothelial cells. $J$. Biol. Chem. 272:1395-1401.

32. Min, W., et al. 1998. The N-terminal domains target TNF receptorassociated factor- 2 to the nucleus and display transcriptional regulatory activity. J. Immunol. 161:319-324.

33. Thorson, J.A., et al. 1998. 14-3-3 proteins are required for maintenance of Raf-1 phosphorylation and kinase activity. Mol. Cell. Biol. 18:5229-5238.

34. Peterson, T., et al. 1999. Opposing effects of reactive oxygen species and cholesterol on endothelial nitric oxide synthase and endothelial cell caveolae. Circ. Res. 85:29-37.

35. De Keulenaer, G.W., et al. 1998. Oscillatory and steady laminar shear stress differentially affect human endothelial redox-state: role of a superoxide-producing NADH oxidase. Circ. Res. 82:1094-1101.

36. Xing, H., Zhang, S., Weinheimer, C., Kovacs, A., and Muslin, A.J. 2000. 14-3-3 proteins block apoptosis and differentially regulate MAPK cascades. EMBO J. 19:349-358. 